\title{
A Regression Analysis and Study of COX-2 Inhibitors Verified by Phylogenetic Tree
}

\author{
Chunduru Madhava Rao \\ Assistant Professor \\ Department of ECE \\ Sir C R Reddy College of Engg \\ Eluru, Andhra Pradesh \\ India
}

\author{
Yesu Babu Adimulam, PhD \\ Professor and HoD, \\ Department of CSE \\ Sir C R Reddy College of Engg \\ Eluru, Andhra Pradesh \\ India
}

\begin{abstract}
Cyclo-oxygenase-2 (COX-2), a rate-limiting enzyme for prostanoid synthesis, is induced during inflammation and participates in inflammation-mediated cytotoxicity. The discovery of two cyclo-oxygenase (COX) isoenzymes, viz. COX-1 and COX-2, has updated the knowledge of non steroidal anti-inflammatory drugs (NSAIDs). The two COX isoenzymes share structural and enzymatic similarities, but are specifically regulated at the molecular level and may be distinguished apart in their functions, although some physiological overlap between them does occur. The major goal in developing selective COX inhibitors is to improve NSAID tolerability. Conventional non steroidal antiinflammatory drugs (NSAIDs) nonspecifically inhibit cyclooxygenase-1 (COX-1), an enzyme critical to normal platelet function, and $\mathrm{COX}-2$ which mediates inflammatory response mechanisms. Celecoxib, the 1, 5-diarylpyrazole compounds was the first launched selective COX-2 inhibitor, and has excellent selectivity and potent anti-inflammatory activity. COX-2 is required for both the constitutive and mitogen-induced $\mathrm{PGE}_{2}$ synthesis. Moreover, over-expression and persistent expression of COX-2 may be influenced by breast tumor hormone status and seem to be a feature of the aggressive, metastaticpheno type. Recent studies have indicated that the relationships between polyunsaturated fatty acid metabolism and carcinogenesis have led to new targets for the design of mechanism based drugs in cancer chemoprevention research. Selective inhibition of COX-2 provided a new class of anti inflammatory, analgesic, and antipyretic drugs with significantly reduced side effects. It has been reported that inhibiting COX-2 could also be an important strategy for preventing or treating a number of cancers. COX-2 selective inhibitors such as celecoxib, rofecoxib and valdecoxib are currently being used to reduce inflammatory response. However, they lack anti-thrombotic activity and hence lead to cardiovascular and renal liabilities apart from gastrointestinal irritation. Therefore, there is still a need to develop more potent COX-2 inhibitors. One of the keys to developing COX-2 selective drugs is the larger active site of COX-2, which makes it possible to make molecules too large to fit into the COX-1 active site but still able to fit the COX-2.
\end{abstract}

\section{Keywords}

COX-2, Phylogenetic tree, Correlation, Inhibitors.

\section{INTRODUCTION}

Cyclo-oxygenase $(\mathrm{COX})$ is a rate-limiting enzyme that converts arachidonic acid to prostaglandins (PGs) and exists in two isoforms, COX-1 and COX-2. Despite the molecular and histological similarities between fetal bone development and fracture healing, inflammation is an early phase of fracture healing that does not occur during development. Cyclo-oxygenase $2(\mathrm{COX}-2)$ is induced at inflammation sites and produces pro inflammatory prostaglandins. Radiographic, histological, and mechanical testing determined that fracture healing failed in rats treated with COX-2-selective NSAIDs (celecoxib and rofecoxib). Normal fracture healing also failed in mice homozygous for a null mutation in the COX-2 gene. This shows that COX-2 activity is necessary for normal fracture healing and confirms that the effects of COX-2selective NSAIDs on fracture healing is caused by inhibition of COX-2 activity and not from a drug side effect. Histological observations suggest that $\mathrm{COX}-2$ is required for normal endochondral ossification during fracture healing. Because mice lacking Cox2 form normal skeletons, our observations indicate that fetal bone development and fracture healing are different and that COX-2 function is specifically essential for fracture healing [1].

\subsection{Differential functions of the COX proteins}

If the only basis for the differences between the COX isoforms was their differential gene expression, then replacing the gene for COX-2 with that for COX-1 should produce no noticeable phenotype. However, "knockin" of the COX-1 gene into the COX-2 locus in mice only partially replenishes the deficit in PGI2 synthesis and fails to fully ameliorate defects in reproductive and renal function associated with COX-2 deletion [2]. These results clearly indicate that COX-1 and COX-2 are not functionally interchangeable at the protein level.

One basis for the results of the COX-1 knock in study may lie in differential coupling between the two COX proteins and downstream syntheses. For example, lack of full restoration of PGI2 synthesis by COX-1 knock in may be due to a failure of coupling between COX-1 with PGI syntheses. Numerous studies support selective isoform association [3 and 4], but much of this work has been done with cells over expressing the relevant enzymes, and no basis for the differential coupling has been advanced. Therefore, confirmation of this hypothesis awaits further investigation.

Differences in substrate specificity between the COX isoforms are not limited to neutral derivatives of 20:4. Indeed, COX-2 has greater capacity to oxygenate a number of polyunsaturated free fatty acids that are poor substrates for COX-1 (Fig. 1) [5]. Since these include v-3 fatty acids, it is possible that the differential use of these lipids by the COX enzymes may help to explain the health benefits of dietary v-3 fatty acids, a hypothesis that remains an intriguing subject for future work [6]. 
A final difference in $\mathrm{COX}$ isoform function that may be of physiologic significance lies in the ability of the aspirintreated enzymes to oxygenate AA. Aspirin inhibits PG formation by both isoforms via covalent modification of Ser530 in the cyclo oxygenase active site. However, COX-2 retains the capacity to oxygenate AA, resulting in the formation of 15R-hydroperoxy-eicosatetraenoic acid instead of PGG2 (34). Through this lipoxygenase-type reaction, aspirin-treated COX-2, together with other lipoxygenases, forms poly-hydroxylated lipids known as aspirin-triggered lipoxins and resolvins. Since these lipids have antiinflammatory activity, their production may help to explain some of the clinical benefits of aspirin [7].

Endocannabinoids

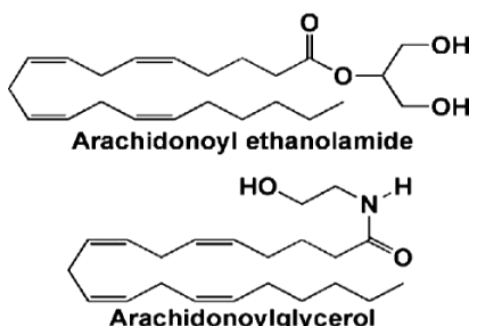

Free Fatty Acids
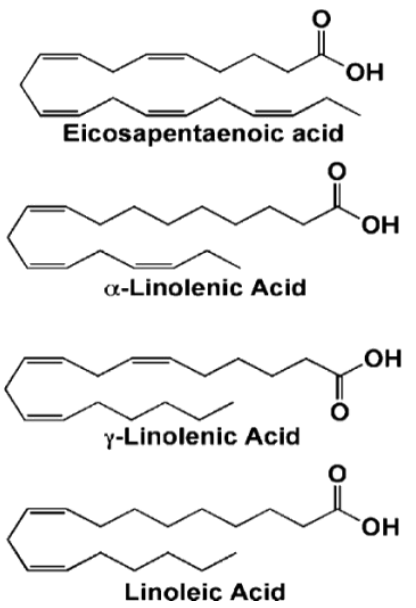

Fig.1: Selective COX-2 substrates. The structures of endocannabi-noids and free fatty acids that are metabolized more efficiently by COX-2 than by COX-1 are shown.

\section{MATERIALS AND METHODS}

\subsection{ISIS Draw}

ISIS Draw is a chemical drawing program similar to Chem raw. When ISIS Draw is started, a window appears with a list of tools down the left hand side of the window, and a list of templates along the top. There are two buttons towards the top left of the window: Molecule and Sketch. To draw chemical structures, Molecule is selected. Using Sketch, one can draw other shapes which may be useful to annotate and beautify diagrams. An image showing 2D structure of a compound is given in Fig.2.

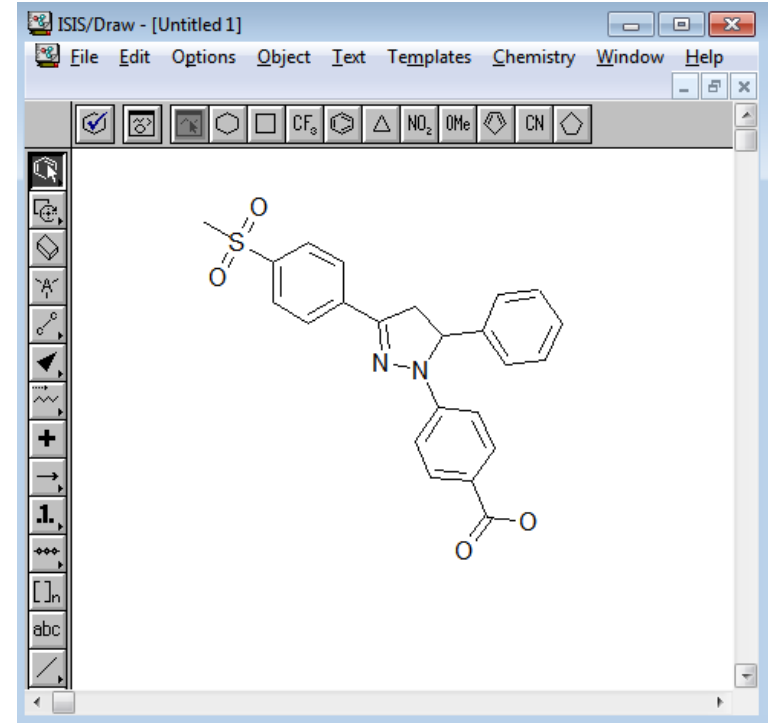

Fig: 2: Screen-shot image of ISIS Draw v3.2 showing compound 8a

\subsection{Tsar Software}

Tsar (Tools for Structure Activity Relationships) is an integrated analysis package used to investigate Quantitative Structure-Activity Relationships. It is intended to provide all the functions required to carry out any investigation, whether in pharmaceutical, agrochemical, toxicological, or any other area of application. It uses a spreadsheet to store and manipulate different types of data, including:

- Molecular descriptions

- $\quad 3 \mathrm{D}$ structures

- Activity data

- Computed data.

\subsection{Correlation}

The Correlation of the dependent and independent variables measures the strength of the relationship between the two. Since our unit of measure may influence the slope of our regression line, we want our correlation to be dimensionless. In general, a positive correlation indicates a strong positive relationship, where an increase in one variable implies an increase in the other variable. A negative correlation indicates that an increase in the first variable implies a decrease in the second variable. A correlation of zero does not necessarily imply there is no relationship between the variables, if the data is non-linear. Often, the Pearson Correlation Coefficient is used to measure correlation, as denoted $r$. Here is the formula:

$$
r=\frac{\sum_{i=1}^{n}\left(x_{i}-\bar{x}\right)\left(y_{i}-\bar{y}\right)}{\sqrt{\sum_{i=1}^{n}\left(x_{i}-\bar{x}\right)^{2}} \sqrt{\sum_{i=1}^{n}\left(y_{i}-\bar{y}\right)^{2}}}
$$

\subsection{Python code: k-means}

The complete python code implementing k-means algorithm is given here.

import math

import random 


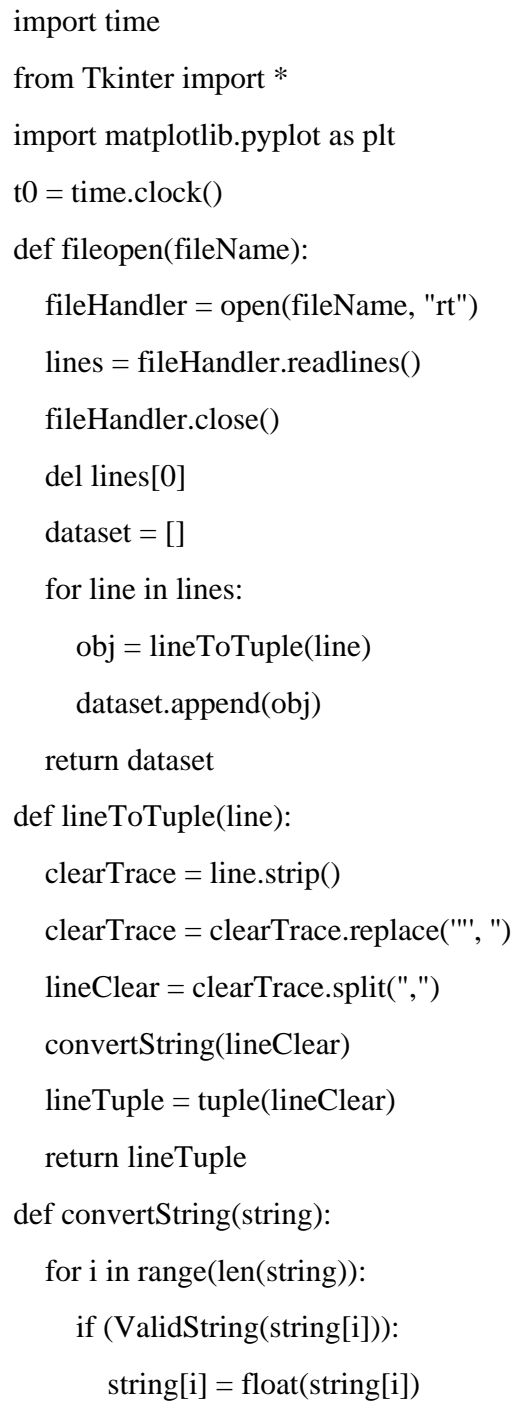

def ValidString(s):

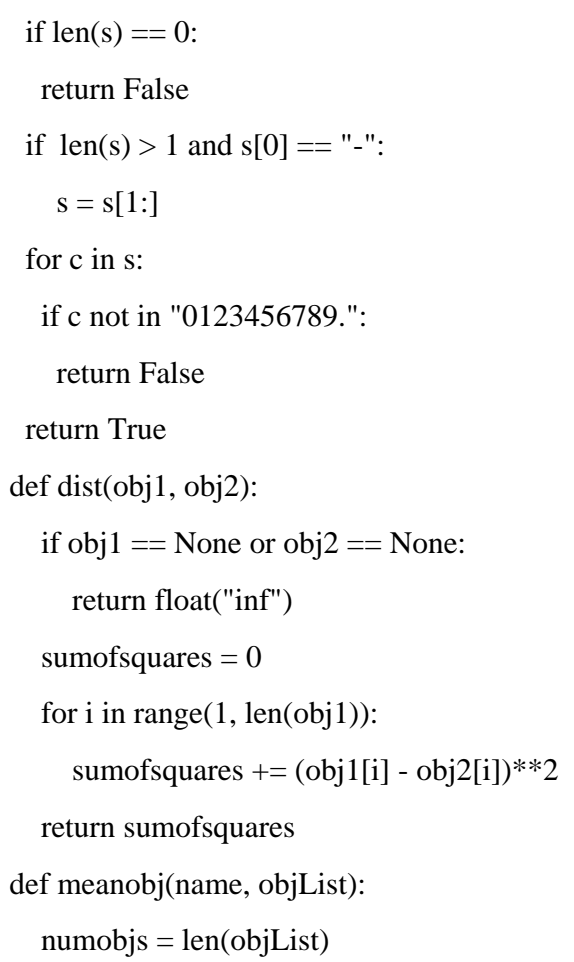

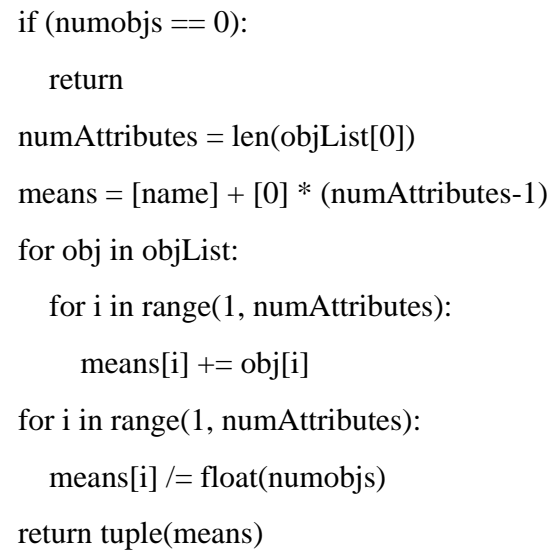

def assign(obj, centroids):

mindist $=\operatorname{dist}(\mathrm{obj}$, centroids $[0])$

mindistIndex $=0$

for $i$ in range(1, len(centroids)):

$\mathrm{d}=\operatorname{dist}(\mathrm{obj}$, centroids[i])

if $(\mathrm{d}<$ mindist $)$ :

mindist $=\mathrm{d}$

mindistIndex $=\mathrm{i}$

return mindistIndex

def createEmptyListOfLists(numSubLists):

string $=[]$

for $\mathrm{i}$ in range(numSubLists):

string.append([])

return string

def assignAll(objs, centroids):

clusters $=$ createEmptyListOfLists(len(centroids))

for obj in objs:

clusterIndex $=\operatorname{assign}(\mathrm{obj}$, centroids)

clusters[clusterIndex].append(obj)

return clusters

def computeCentroids(clusters):

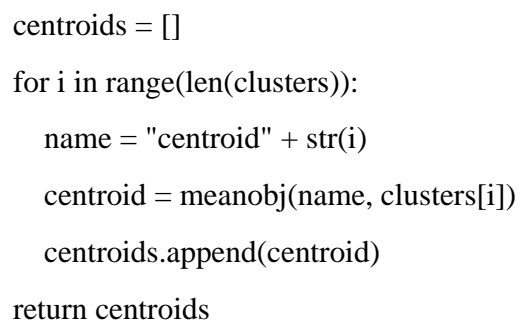

def kmeans(objs, $\mathrm{k}$, animation=False, initCentroids=None):

result $=\{\}$

if (initCentroids $==$ None or len $($ initCentroids $)<\mathrm{k}$ ):

random.seed(time.time())

centroids $=$ random.sample $($ objs, $\mathrm{k})$

else: 


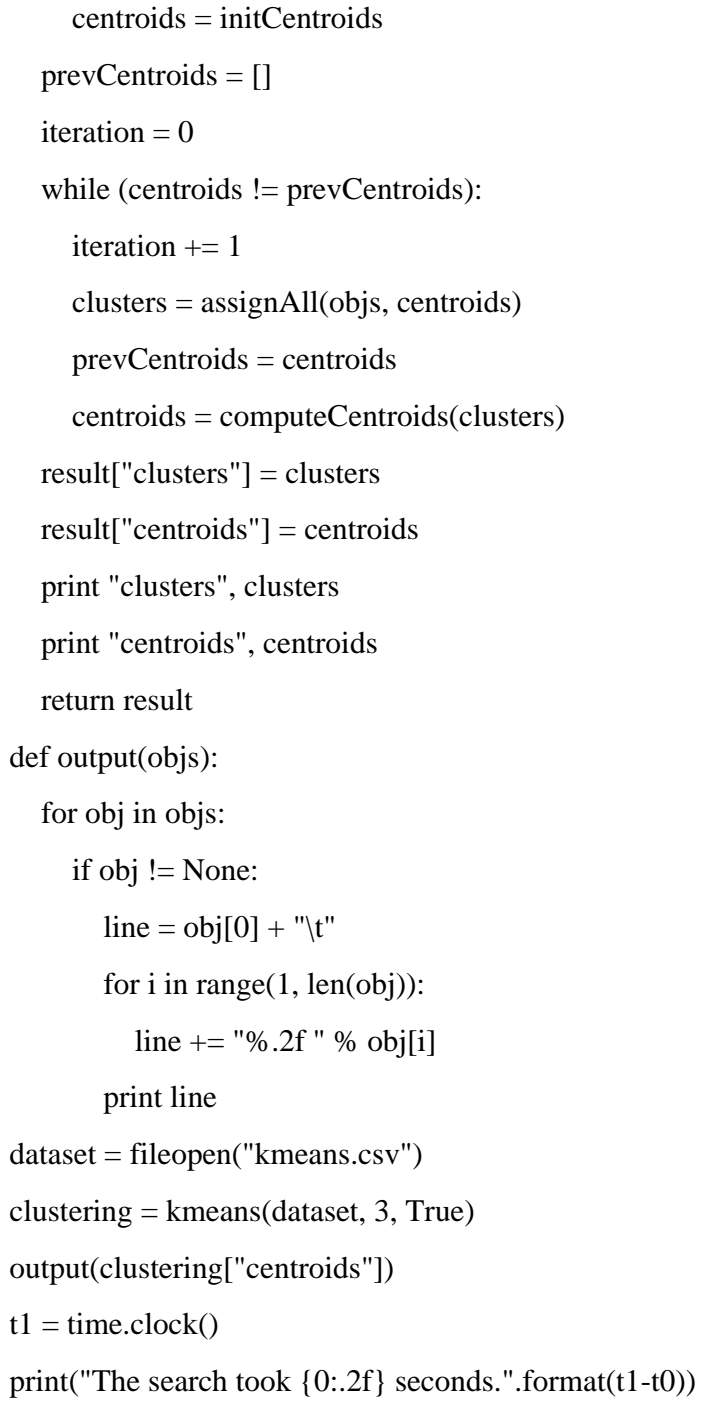

\section{RESULTS AND DISCUSSION}

Multivariate regression analysis using python program resulted in few influential parameters displayed significant positive and negative contribution towards biological activity of COX-2 inhibitors.

\subsection{Clustering Analysis}

The k-means clustering algorithm is popular because it can be applied to relatively large sets of data. The user specifies the number of clusters to be found. The algorithm then separates the data into spherical clusters by finding a set of cluster centers, assigning each observation to a cluster, determining new cluster centers, and repeating this process.

A modified k-means python program was written and implemented to evaluate the clusters and assessing the probability of designed compounds to appear in a particular cluster thereby confirming the similarity of such compounds to appear in a cluster of approved drugs.

The data file is formatted as a comma delimited file .csv and the file was called from python. Further clustering code was implemented to perform k-means clusters on the dataset by calculating number of clusters for given centroids. The number of centroids can be changed depending on the data being clustered. In this case, 3 centroids are considered. Now, the k-means algorithm was implemented by finding distances between objects taken for the study. Once distances between objects are calculated, the sum of squares for the two objects from the centroid is calculated. Following this, means are calculated for all objects which are nearer to each other making it to study all possible objects and the List of such objects will be created by the program to iterate the process. Centroids are assigned and the distance is calculated from each centroid to the objects and clusters are identified. To find out better clusters, a minimum distance is calculated from each object to the nearest assigned centroid. Once each object is assigned to its nearest centroid, then the distances are recalculated by adjusting the centroids in such a way that the objects should have the shortest possible distance with the centroid. Likewise, a cluster index is created and appended to the objects. Finally, the $\mathrm{k}$ in k-means is calculated by randomly selecting $\mathrm{k}$ initial centroids.

\section{Algorithm: K-Means}

1. Initialization: choose $\mathrm{k}$ initial centroids arbitrarily (or randomly).

2. Assign each data point to the centroid that is closer to it.

3. Compute the distance between the centroids and objects using the Euclidean Distance equation

4. Update all the centroids. The new centroid of a cluster is the mean of all the points within that cluster.

5. Repeat points 2 and 3 until the new centroids are the same as the previous centroids.

K-means suffers from drawback on the number of clusters $\mathrm{k}$ as an input parameter. This is because of an inappropriate choice of $\mathrm{k}$ which might yield spurious results. Hence, it is always an important task to run diagnostic checks when using k-means clustering to determine the number of clusters in the given dataset. Moreover, applying k-means with values ranging from $\mathrm{k}=2,3,4$ or 5 depends on the number of objects in the dataset and to avoid expected clusters of similar size so that the assignment of objects to the nearest cluster center or centroid will result in correct clusters. Therefore, an attempt has been made to cluster dataset using different $\mathrm{k}$ value ranges. The first step in $\mathrm{k}$-means algorithm is to divide the given data set into user defined number of clusters. The initial choice of $\mathrm{k}$ in $\mathrm{k}$-means is an interpretive decision and successive runs should be performed to obtain an optimized division of data for any chosen $\mathrm{k}$ value. A prior knowledge on the data structure would result in more appropriate clusters. However, as the dimensionality of the data increases, it becomes increasingly difficult to determine a proper value for $\mathrm{k}[8]$.

\subsection{FIT Kubinyi Function}

All the three models passed the conditions for validation sets. Further, to define the statistical quality of activity prediction, the number of variables that enter in a regression equation are compared by using FIT Kubinyi function (Eq. 10), a criteria closely related to $\mathrm{F}$ value was proven to be useful [9].

Eq. 10:

FIT $=R^{2}(n-k-1) /\left(n+k^{2}\right)\left(1-R^{2}\right)$

Where $\mathrm{n}$ is the number of compounds in training set and $\mathrm{k}$ is the number of variables in the regression equation.

The main feature of the $F$ value is its sensitivity to changes in $\mathrm{k}$, if $\mathrm{k}$ is small and its lower sensitivity if $\mathrm{k}$ is large. The FIT criterion has a low sensitivity towards changes in $\mathrm{k}$ values, as 
long as they are small numbers, and a substantially increasing sensitivity for large $\mathrm{k}$ values [10]. The best model will be the one that possess a high value of this function. Hence, regression models with 12, 9 and 11 variables are generated (Table 1) to choose the best among them.

Table 1: Statistical parameters of the regression models obtained for 12, 9 and 11 variables.

\begin{tabular}{|l|l|l|l|l|}
\hline Var $^{\mathbf{a}}$ & $\boldsymbol{r}^{2}$ & $\boldsymbol{k}$ & FIT & Eq No. \\
\hline 12 & 0.782 & 12 & $\mathbf{0 . 7 9 9}$ & 10 \\
\hline 9 & 0.645 & 9 & 0.627 & 11 \\
\hline 11 & 0.741 & 11 & 0.735 & 12 \\
\hline
\end{tabular}

\subsection{Screening non-tested compounds from literature}

Based on the above, it can be stated that the proposed model due to its high predictive ability can be utilized as an alternative aid to the costly and time consuming experiments for recognizing and determining compounds with high COX-2 binding affinity. The method can also be used to screen similar repertoire of compounds reported in various literature sources or chemical compound libraries to identify new potentially active compounds.

Hence, a group of new derivatives from literature are subjected to screening utilizing the produced model. We, therefore, searched for compounds with pyrazoles, pyrazolines, or other pyrazole derivatives in Archives of organic chemistry journal [11]. Therefore, a set of 32 compounds with pyrazole ring as main nucleus was selected from a published review paper [12]. The structures are devoid of biological activity against COX-2, however, these pyrazole compounds are known to inhibit certain disease causing protein targets, for example, Factor $\mathrm{Xa}$, IXa, platelet aggregation inhibition, antidepressant, voltage-gated sodium channel blockers etc. All compounds screened for this study are provided with compound numbers as given in reference article (Table 2)

Table 1: Compounds along with predicted activity data after applying regression model on this 32 compound dataset

\begin{tabular}{|l|l|l|}
\hline $\begin{array}{l}\text { Molecule } \\
\text { No. }\end{array}$ & $\begin{array}{l}\text { Predicted } \\
\text { Activity } \\
\left(\mathbf{l o g 1 / I}_{\mathbf{5 0}}\right)\end{array}$ & $\begin{array}{l}\text { Calculated } \\
\mathbf{I C}_{\mathbf{5 0}}(\mathbf{u M})\end{array}$ \\
\hline $28 . \mathrm{mol}$ & -0.6172 & 4.1420 \\
\hline $35 . \mathrm{mol}$ & -1.1829 & 15.2383 \\
\hline $37 . \mathrm{mol}$ & -2.0143 & 103.3463 \\
\hline $38 . \mathrm{mol}$ & 0.5860 & 0.2594 \\
\hline $42 . \mathrm{mol}$ & -2.2016 & 159.0788 \\
\hline $52 . \mathrm{mol}$ & -2.2864 & 193.3873 \\
\hline $57 . \mathrm{mol}$ & -1.0800 & 12.0230 \\
\hline $58 . \mathrm{mol}$ & 0.7703 & 0.1697 \\
\hline $62 . \mathrm{mol}$ & 0.1038 & 0.7874 \\
\hline $64 . \mathrm{mol}$ & -2.3705 & 234.7178 \\
\hline $65 . \mathrm{mol}$ & -2.1758 & 149.9144 \\
\hline $66 . \mathrm{mol}$ & -1.5239 & 33.4115 \\
\hline $67 . \mathrm{mol}$ & 0.9942 & 0.1013 \\
\hline
\end{tabular}

\begin{tabular}{|l|l|l|}
\hline 68.mol & $\mathbf{1 . 9 1 9 1}$ & $\mathbf{0 . 0 1 2 0}$ \\
\hline 69.mol & 2.1406 & 0.0072 \\
\hline 70.mol & 0.6351 & 0.2317 \\
\hline 72.mol & $\mathbf{1 . 8 5 7 2}$ & $\mathbf{0 . 0 1 3 9}$ \\
\hline 73a.mol & $\mathbf{1 . 1 7 7 4}$ & $\mathbf{0 . 0 6 6 5}$ \\
\hline 73b.mol & $\mathbf{2 . 8 8 0 3}$ & $\mathbf{0 . 0 0 1 3}$ \\
\hline 73c.mol & $\mathbf{1 . 8 0 2 2}$ & $\mathbf{0 . 0 1 5 8}$ \\
\hline 74a.mol & 1.2406 & 0.0575 \\
\hline 74b.mol & -0.1164 & 1.3074 \\
\hline 74c.mol & 1.9006 & 0.0126 \\
\hline 75.mol & -0.5985 & 3.9675 \\
\hline 76a.mol & -0.3190 & 2.0843 \\
\hline 76b.mol & -1.7174 & 52.1630 \\
\hline 77.mol & 1.4140 & 0.0385 \\
\hline 78.mol & -1.5899 & 38.8932 \\
\hline 79.mol & -2.0934 & 123.9857 \\
\hline $80 . \mathrm{mol}$ & -3.6364 & 4329.3848 \\
\hline $81 . \mathrm{mol}$ & -2.8340 & 682.3371 \\
\hline $90 . \mathrm{mol}$ & -0.8823 & 7.6259 \\
\hline Celecoxib & $\mathbf{0 . 2 4 7 2}$ & $\mathbf{0 . 5 6 6 0}$ \\
\hline
\end{tabular}

\section{CONCLUSIONS}

Cyclooxyenase-2 is an enzyme which is mainly used in the treatment of various disorders such as breast cancer, atherosclerosis etc. Cyclooxygenase-2 (COX-2) enzyme binds to arachidonic acid and releases metabolites that are used to induce pain and inflammation. COX-2 selective inhibitors such as celecoxib, rofecoxib and valdecoxib are currently being used to reduce inflammatory response. However, they lack anti-thrombotic activity and hence lead to cardiovascular and renal liabilities apart from gastrointestinal irritation. Therefore, there is still a need to develop more potent COX-2 inhibitors.

Further, applying FIT Kubinyi function on the models resulted in model with 12 variables as the best model. Hence, designing or screening compound libraries for new compounds or analogs with possible H-bond acceptor and donor substitutions, and increase in number of Fluorine atoms and decrease in molecular weight, logp and rotatable bonds on the molecule with concomitant increase in dipole. Lipole and molar refractivity would enhance inhibitory activity against COX-2.

Finally, a phylogenetic tree was presented to know the branches on which these novel compounds appear and hence can be used to further extend the study towards identifying more statistical parameters that would substantiate the identification of entirely novel, potent COX-2 inhibitors.

\section{REFERENCES}

[1] Developmental expression of the cyclo-oxygenase-1 and cyclo-oxygenase-2 genes in the peri-implantation mouse uterus and their differential regulation by the blastocyst and ovarian steroids \{Chakraborty, SK Das, J Wang, and SK Dey, \}, Journal of Molecular Endocrinology, Vol 16, Issue 2, 107-122.

[2] Yu, Y., J. Fan, Y. Hui, C. A. Rouzer, L. J. Marnett, A. J. Klein-Szanto, A. FitzGerald, and C. D. Funk. 2007. Targeted cyclooxygenase gene (ptgs) exchange reveals discriminant isoform functionality. Biol. Chem. 282: 1498-1506.

[3] Ueno, N., Y. Takegoshi, D. Kamei, I. Kudo, and M. Murakami. 2005. Coupling between cyclooxygenases 
and terminal prostanoid syn-thases. Biochem. Biophys. Res. Commun. 338: 70-76.

[4] Smith, W. L., and R. Langenbach. 2001. Why there are two cyclo-oxygenase isozymes. J. Clin. Invest. 107: 1491-1495.

[5] Laneuville, O., D. K. Breuer, N. Xu, Z. H. Huang, D. A. Gage, J. T. Watson, M. Lagarde, D. L. DeWitt, and W. L. Smith. 1995. Fatty acid substrate specificites of human prostaglandin-endoperoxide $\mathrm{H}$ synthase- 1 and -2 . Formation of 12-hydroxy-(9Z,13E/Z,15Z)-octadeca trienoic acids from a-linolenic acid. J. Biol. Chem. 270: 19330-19336.

[6] Smith, W. L. 2005. Cyclooxygenases, peroxide tone and the allure of fish oil. Curr. Opin. Cell Biol. 17: 174-182.

[7] Serhan, C. N., N. Chiang, and T. E. Van Dyke. 2008. Resolving in-flammation: dual anti-inflammatory and pro-resolution lipid mediators. Nat. Rev. Immunol. 8: 349-361.
[8] Jonathan Baarsch and M. Emre Celebi "Investigation of Internal Validity Measures for K-Means Clustering" in Proceedings of the International Multi conference of Engineers and Computer Scientists 2012 Vol 1, IMCES 2012, March 14-16 2012, HongKong.

[9] Kubinyi H. Variable selection in QSAR studies. II. A Highly Efficient Combination of Systematic Search and Evolution. Quant. Struct. Act. Relat. 13, 1994, 393-401.

[10] Kubinyi H. Variable selection in QSAR studies. I. An evolutionary algorithm. Quant. Struct. Act. Relat. 13, 1994, 285-294.

[11] www.arkat-usa.org

[12] Ruth Pérez-Fernández, Pilar Goya, and José Elguero. A review of recent progress (2002-2012) on the biological activities of pyrazoles. ARKIVOC 2014 (ii) 233-293. 